\title{
Effects of different exercises on the growth plate in young growing mice.
}

\author{
Eriko Mizuno ${ }^{1}$, Yoshio Wakimoto ${ }^{1}$, Masato Nomura ${ }^{1}$, Yuta Kohara ${ }^{1}$, Shunsuke Shimaya ${ }^{1}$, Ryota \\ Suzuki $^{1}$, Hideki Moriyama ${ }^{2 *}$ \\ ${ }^{1}$ Department of Rehabilitation Science, Graduate School of Health Sciences, Kobe University, Hyogo, Japan \\ ${ }^{2}$ Life and Medical Sciences Area, Health Sciences Discipline, Kobe University, Hyogo, Japan
}

\begin{abstract}
Background and objective: Chondrocytes in the growth plate are a major player in the process of endochondral ossification through from proliferation to hypertrophy, leading to the longitudinal growth of the skeleton. Exercise promotes the longitudinal growth, whereas excessive exercise inhibits bone growth. The exercise condition is defined as a combination of intensity, frequency, and duration; and what these combinations leading to the best for the bone growth remain unclear. Our objective was to determine what combination of different exercise conditions lead to the best for a favorable response in the growth plate.

Methods: A total of 27 male young mice were divided into control group and 8 treadmill exercise groups; high-intensity (running), high-frequency (every day), and long-duration (60 min); high-intensity, high-frequency, and short-duration (15 min); high-intensity, low-frequency (Once in $3 \mathrm{~d}$ ), and longduration; high-intensity, low-frequency, and short-duration; low-intensity (walking), high-frequency, and long-duration; low-intensity, high-frequency, and short-duration; low-intensity, low-frequency, and long-duration; low-intensity, low-frequency, and short-duration. We quantified histologic characteristics in the growth plate after exercise.

Results: The thickness of the growth plate, cell proliferation, and the number of hypertrophic chondrocytes in the growth plate increased in the high-intensity exercise compared with the lowintensity; further, osteoclasts in the primary spongiosa increased by high-intensity and low-frequency and long-duration exercise.

Conclusion: Our results suggest that chondrocyte activity and bone metabolism promote in highintensity and low-frequency and long-duration in young mice. These findings may shed light on the underlying of the promotion of the longitudinal bone growth by exercise.
\end{abstract}

Keywords: Exercise, Bone growth, Growth plate.

Accepted on May 18, 2018

\section{Introduction}

Skeletal maturity including the increase in bone length and bone mass occurs rapidly during growth period [1]. Especially, the increase in bone length is the index of growth as stature and is affected by mechanical stresses such as physical exercise [2]. Therefore, the mechanical stress related to exercise promotes the bone growth.

The growth plate chondrocytes are a major player in the process of endochondral ossification through from proliferation to hypertrophy that contributes to the longitudinal growth of the skeleton [3-5]. An animal study has reported that treadmill exercise increases the mRNA expression of type $\mathrm{X}$ collagen synthesized by hypertrophic chondrocytes in the increased growth plate and decreases the thickness of the growth plate (the proliferative and hypertrophic zones) [6,7]. Also, swimming did not change the number of hypertrophic chondrocytes, whereas increased the thickness of the growth plate and the number of proliferative chondrocyte [8]. Owing to the discrepancies among these results so far, the response in the growth plate is controversial and remains elusive, although the longitudinal growth of the bone is greatly affected by exercise. These controversial results can be related to particularly exercise conditions as well as ages or exercise modes.

The exercise condition is defined as a combination of intensity, frequency, and duration [9]. In a growing child, exercise with a more than 3-fold higher impact loading of body weight heavily influences the bone compared with swimming and highintensity exercise increases the bone mass and strength [10-13]. On the other hand, children involved in high-intensity sports are more susceptible to trauma in the growth plate and therefore the training program with low intensity and large quantity is now recommended [14,15]. Taken together, moderate exercise has a favorable effect on the bone growth, 
but excessive exercise inhibits the bone growth and leads to destruction [3]. However, what combination of intensity, frequency, and duration leading to the best for the bone growth is still poorly understood.

Our objective was to determine what combination of different exercise conditions leads to the best for a cell response in the growth plate. To reach our goal, we quantified histologic characteristics in the growth plate after different treadmill exercises in a growing mouse.

\section{Materials and Methods}

\section{Experimental design}

All experimental procedures were examined by the Institutional Animal Care and Use Committee, approved by the president of Kobe University, and performed according to the Kobe University Animal Experimentation Regulations. A total of 27 male C57BL/6J mice (7 weeks old, 19-24 g mean body weight, Japan SLC Inc., Japan) were used for this study. The 7 weeks old of a mouse corresponds to the growth period [16]. Animals were housed in standard cages under a $12 \mathrm{~h}$ dark/light cycle at a constant temperature of $22 \pm 1{ }^{\circ} \mathrm{C}$ and allowed free access to standard food and water. These mice were randomly divided into a control group and 8 exercise groups. The right and left hind legs of each animal served as different samples, and 4 out of 6 legs in each group were randomly selected. Total 36 tibias were assessed by histological, immunohistochemical, and histomorphometric analyses.

\section{Exercise protocol}

A treadmill device (MK-680, Muromachi Kikai Co, Ltd., and Japan) was used to provide an exercise load to the mice. Mice were randomly divided into following 9 groups: control (no exercise); high-intensity (18 $\mathrm{m} / \mathrm{min})$, high-frequency (every day), and long-duration (60 $\mathrm{min} / \mathrm{d})$ (HHL); high-intensity, high-frequency, and short-duration (15 min/d) (HHS); highintensity, low-frequency (Once in 3 d) and long-duration (HLL); high-intensity, low-frequency, and short-duration (HLS); low-intensity ( $8 \mathrm{~m} / \mathrm{min})$, high-frequency, and longduration (LHL); low-intensity, high-frequency, and shortduration (LHS); low-intensity, low-frequency, and longduration (LLL); low-intensity, low-frequency, and shortduration (LLS) (Table 1). Each group consisted of three animals. The speed of $18 \mathrm{~m} / \mathrm{min}$ and $8 \mathrm{~m} / \mathrm{min}$ corresponds to running and walking exercise for mice, respectively $[17,18]$. All mice were trained on the treadmill once a day for 4 weeks. This treadmill training had no effect on healthy and physically active of mice, as confirmed by others [19].

Table 1. Groups and exercise protocol.

\begin{tabular}{|c|c|c|c|c|c|}
\hline \multicolumn{3}{|c|}{ Group } & \multicolumn{3}{|c|}{ Exercise protocols } \\
\hline Intensity & Frequency & Duration & Intensity & Frequency & Duration \\
\hline \multirow{4}{*}{ High intensity } & \multirow{2}{*}{ High frequency } & Long duration (HHL) & \multirow{4}{*}{$18 \mathrm{~m} / \mathrm{min}$} & \multirow{2}{*}{ Everyday } & $60 \mathrm{~min}$ \\
\hline & & Short duration (HHS) & & & $15 \mathrm{~min}$ \\
\hline & \multirow{2}{*}{ Low frequency } & Long duration (HLL) & & \multirow{2}{*}{ Once in $3 d$} & $60 \mathrm{~min}$ \\
\hline & & Short duration (HLS) & & & $15 \mathrm{~min}$ \\
\hline \multirow{4}{*}{ Low intensity } & \multirow{2}{*}{ High frequency } & Long duration (LHL) & \multirow{4}{*}{$8 \mathrm{~m} / \mathrm{min}$} & \multirow{2}{*}{ Everyday } & $60 \mathrm{~min}$ \\
\hline & & Short duration (LHS) & & & $15 \mathrm{~min}$ \\
\hline & \multirow{2}{*}{ Low frequency } & Long duration (LLL) & & \multirow{2}{*}{ Once in $3 \mathrm{~d}$} & $60 \mathrm{~min}$ \\
\hline & & Short duration (LLS) & & & $15 \mathrm{~min}$ \\
\hline
\end{tabular}

Control

\section{Tissue preparation for histology}

We prepared un-decalcified frozen sections as previously described in the literature [20]. All mice were sacrificed by exsanguination under anaesthesia and tibias were harvested at the end of the experimental period. The samples were then immediately freeze-embedded with 5\% carboxymethyl cellulose gel. Blocks were cut into slices, and 5- $\mu \mathrm{m}$ frontal sections of the proximal tibia were prepared.

\section{Histological and histomorphometric analyses}

Frozen sections were stained with safranin O/fast green, alkaline phosphatase (ALP) (Sigma387A-1KT, Sigma, Japan), and tartrate-resistant acid phosphatase (TRAP) (Sigma387A-1KT, Sigma, Japan). They were captured with a light microscope (BX-53, Olympus, Japan) at a magnification of $4 \mathrm{X}$ and 20X. ALP and TRAP staining were counterstained with eosin or alcian blue, respectively.

The thickness of the growth plate was measured on digitized images of histological sections stained with safranin $\mathrm{O} /$ fast green. Briefly, the areas of the growth plate and the length of growth plate from the medial edge to the lateral edge were measured separately with Adobe Photoshop CS (Adobe Systems Inc., Japan). The thickness of the growth plate was calculated by dividing the area by the length. 
We measured the ALP and TRAP activity area on digitized images of histological sections. Briefly, ALP and TRAPstained histological images were split into separate channels, and these positive cells at the entire epiphysis were identified on the red and blue channel grayscale images respectively by a certain threshold with Image J 1.50 (National Institutes of Health, Bethesda, MD, USA). Then, the ALP and TRAP activity area per total area was calculated, and the mean area for each specimen was derived by averaging measurements from different 3 regions.

\section{Immunohistochemical analysis and histomorphometric analysis}

The frozen sections were incubated with rabbit monoclonal anti-proliferating cell nuclear antigen (PCNA) (diluted 1:1000, \#13110, Cell Signaling Technology Japan, Japan) or rabbit polyclonal anti-Type X collagen (diluted 1:10000, LB-0092, LSL, Japan) antibodies. Subsequent reaction was made by streptavidin-biotin-peroxidase complex technique with an Elite $\mathrm{ABC}$ kit (diluted 1:50, PK-6100, Vector Laboratories, USA). Color was then developed with 3, 3'-diaminobenzidine tetrahydrochloride (K3466, Dako Japan, and Japan). The sections were finally counterstained with hematoxylin, washed in water, and cover slipped. The section of PCNA staining and type $\mathrm{X}$ collagen staining were captured with a light microscope (BX-53) at a magnification of $40 \mathrm{X}$ and $20 \mathrm{X}$, respectively. Then, the standardized rectangular field $\left(0.09 \mathrm{~mm}^{2}\right.$ for PCNA or $0.37 \mathrm{~mm}^{2}$ for type $\mathrm{X}$ collagen) was superimposed over the histologic sections. We counted manually these immunepositive cells within the rectangular field at different 3 regions and calculated the mean number.

\section{Statistical analyses}

Results for the thickness of growth plate, PCNA or collagen type $\mathrm{X}$ positive cell numbers in the growth plate, and activity area of ALP or TRAP in the primary spongiosa were analyzed statistically with JMP 7 (IBM Japan, Tokyo, Japan). Normality of distribution was assessed with the Shapiro-Wilk test. When normality was observed in all assays, the results were compared among all groups with ANOVA followed by LSD significant difference test. An alpha of less than 0.05 was chosen as the significance level for all statistical analyses. All values in the text and figures are presented as mean \pm Standard Error (SE).

\section{Results}

\section{Thickness of the growth plate}

Histologically, growth plates remained open continuously without partial closure in all groups (Figure 1A). Cartilage matrix staining to safranin as a reflection of proteoglycan content showed no difference among the groups. The thickness of the growth plate significantly increased in the HLS, HLL and HLS groups, when compared to the control group (Figure 1B).

\section{Localization of PCNA positive cell}

We observed the localization of PCNA as a marker of cell proliferation. The PCNA-positive cells distributed in the proliferative zone of the growth plate in all groups (Figure 2A); The number of these cells significantly increased in 3 highintensity groups compared with the LHL and LLS groups, and in the HHS, HLL, and HLS groups compared with LHS group (Figure 2B). However, there were no differences between all exercise groups and the control group.
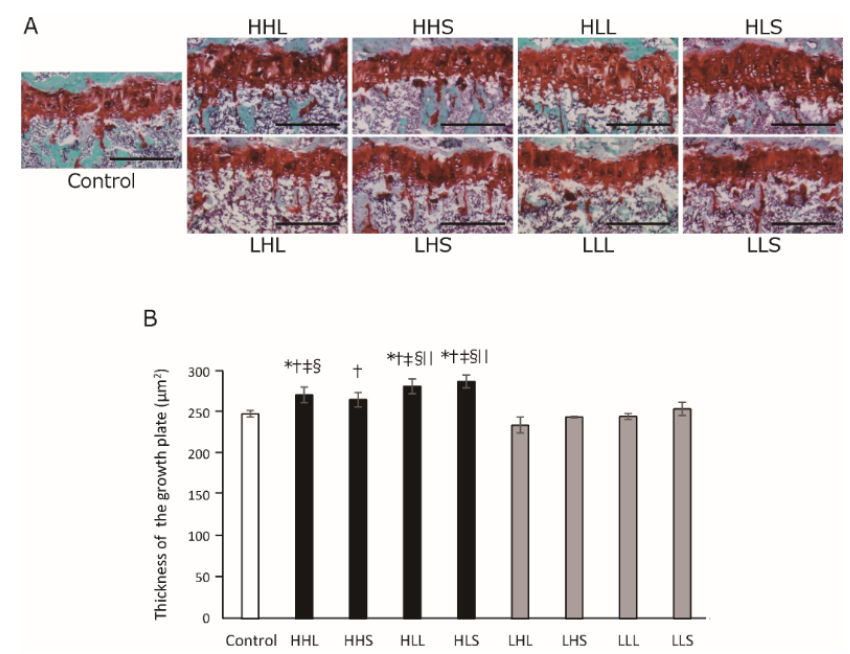

Figure 1. (A) Representative image of frontal section of proximal tibia stained with safranin O/first green. Scale bars are $200 \mu m ;(B)$ The thickness of the growth plate. Data are presented as mean $\pm S E$. Statistical differences are shown as follows: ${ }^{*} P<0.05$ vs. control; ${ }^{\dagger} P<0.05$ vs. LHL; ${ }^{t} P<0.05$ vs. LHS; ${ }^{\S} P<0.05$ vs. LLL; $\| P<0.05$ vs. $L L S$.
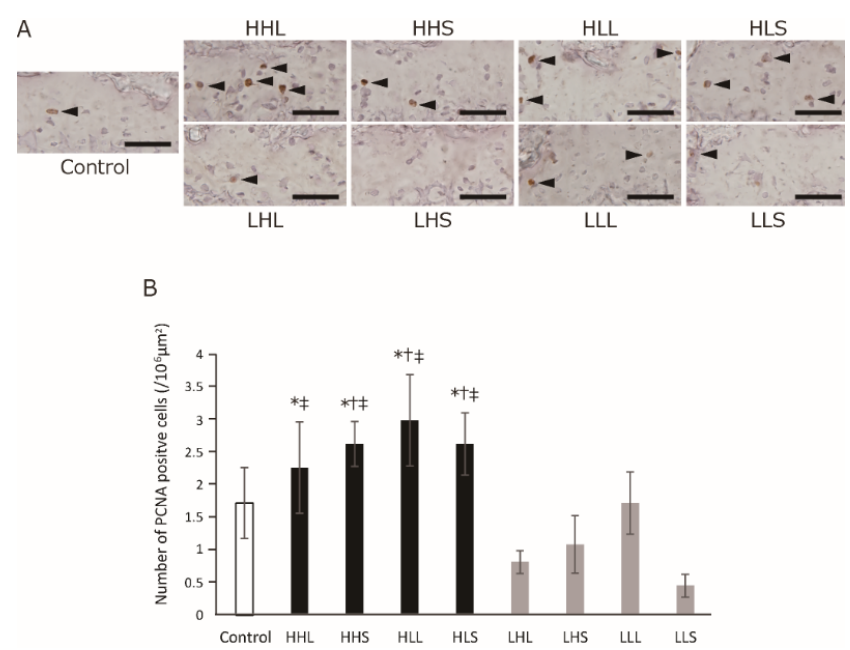

Figure 2. (A) Localization of PCNA positive cells by immunostaining. Positive cells are represented by arrowheads. Scale bar is $20 \mu \mathrm{m}$; (B) PCNA positive cells number. Data are presented as mean $\pm S E$. Statistical differences are shown as follows: ${ }^{*} P<0.05$ vs. LHL; ${ }^{\dagger} P<0.05$ vs. LHS; ${ }^{\ddagger} P<0.05$ vs. LLS.

\section{Localization of type $X$ collagen}

The type X collagen-positive cells, hypertrophic chondrocytes, distributed in the calcification zone of the growth plate in all 
groups (Figure 3A); The numbers of these cells significantly increased in the HHL and HLL groups compared with the LHL group, and in the HLL and HLS groups compared with the LHS and LLL groups (Figure 3B), although no statistical difference was found when comparing the control group.
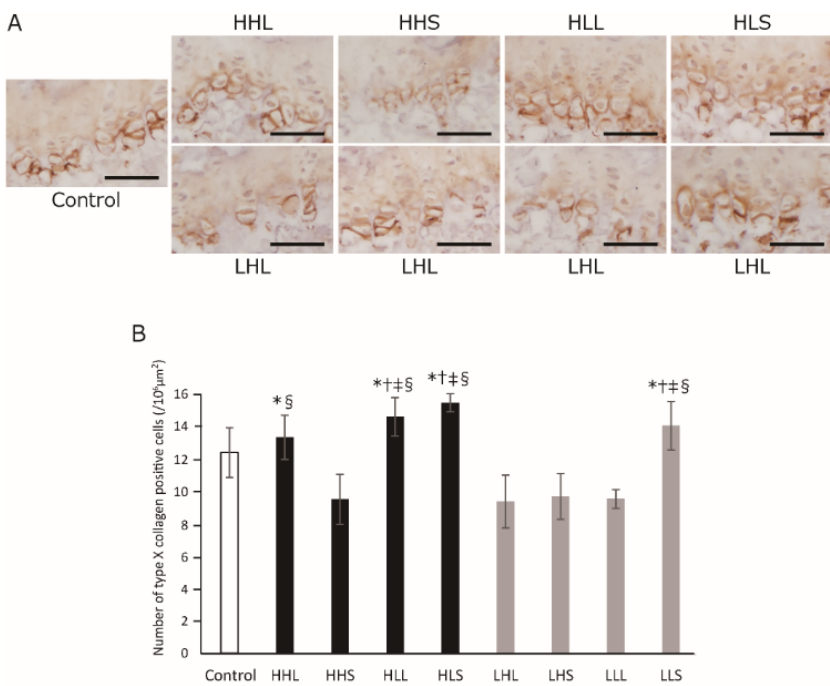

Figure 3. (A) Localization of type $X$ collagen positive cells by immunostaining. Scale bar is $20 \mu m$; (B) Type X collagen positive cells number. Data are presented as mean \pm SE. Statistical differences are shown as follows: ${ }^{*} P<0.05$ vs. LHL; ${ }^{\dagger} P<0.05$ vs. LHS; ${ }^{t} P<0.05$ vs. $L L L ;{ }^{\S} P<0.05$ vs. HHS
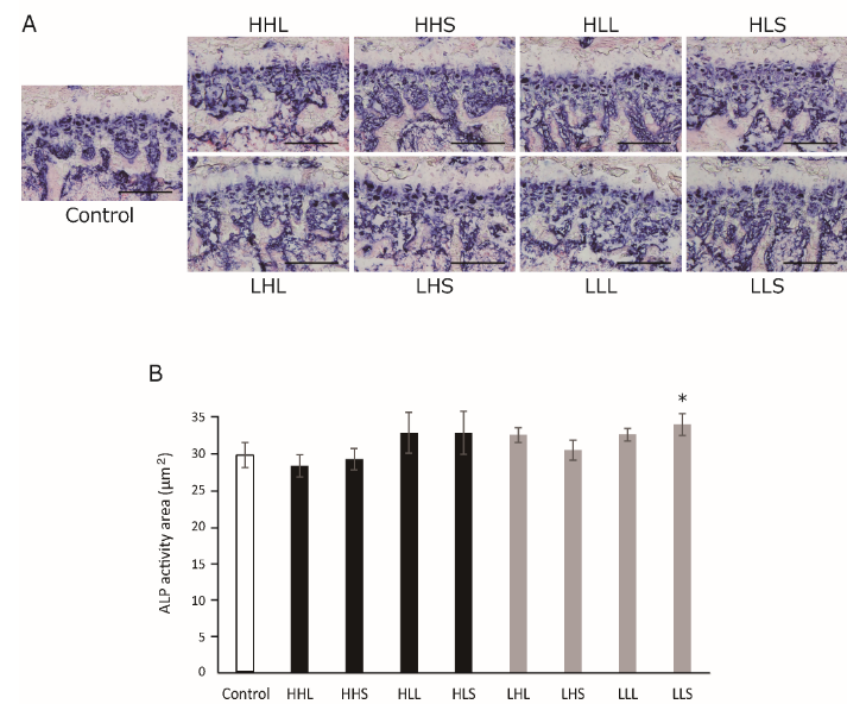

Figure 4. (A) Representative image stained with ALP/eosin. Scale bar is $100 \mu \mathrm{m}$; (B) ALP activity area. Data are presented as mean $\pm S E$. Statistical differences are shown as follows: ${ }^{*} P<0.05$ vs. HHL.

\section{Localization of the ALP and TRAP activity}

ALP-positive nucleated cells attached to the bone were scored as osteoblasts, and TRAP-positive multinucleated cells attached to the bone were scored as osteoclasts. In all groups, osteoblasts distributed from just under the growth plate to the primary spongiosa and at the trabecular wall (Figure 4A). The ALP-activity area in the primary spongiosa of all exercise groups was not significant difference from that of the control group (Figure 4B). Similar to osteoblasts, osteoclasts were observed strongly from just under the growth plate to the primary spongiosa and at the trabecular in all groups (Figure 5A). Also, TRAP-activity area of the primary spongiosa was significantly increased in the HLL and LLS groups compared with the control group (Figure 5B).
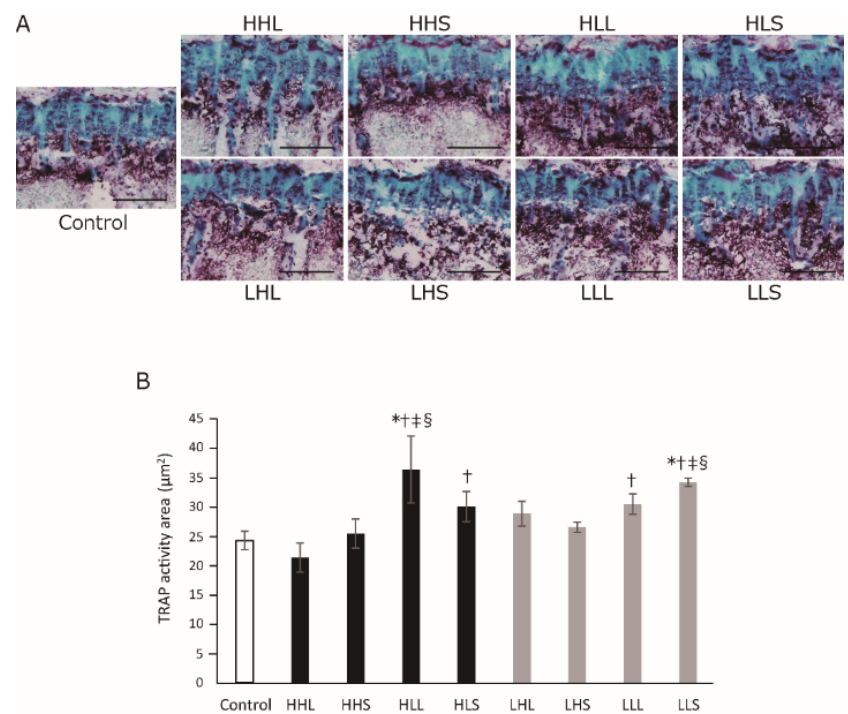

Figure 5. (A) Representative image stained with TRAP/alcian blue. Scale bar is $100 \mu \mathrm{m}$; (B) TRAP activity area. Data are presented as mean $\pm S E$. Statistical differences are shown as follows: ${ }^{*} P<0.05 \mathrm{vs}$. control; ${ }^{\dagger} P<0.05$ vs. HHL; ${ }^{t} P<0.05$ vs. HHS; ${ }^{\S} P<0.05$ vs. LHS.

\section{Discussion}

We quantified histologic characteristics in the tibial growth plate of a growing mouse to determine what combination of intensity, frequency, and duration leading to the best for the longitudinal growth of the bone. As a result, the thickness of the growth plate, cell proliferation, and the number of hypertrophic chondrocytes in the growth plate more increased in the high-intensity exercise than that in the low-intensity. Furthermore, osteoclasts in the primary spongiosa increased by high-intensity and low-frequency and long-duration exercise. These findings suggest that the cell activity and bone metabolism from the growth plate to primary spongiosa promoted by high-intensity exercise in addition to lowfrequency and long-duration.

Generally, the growth plate is comprised of the resting zone, the proliferative zone, the hypertrophic zone, and the calcification zone [3]. Changes in the thickness of the growth plate by exercise result from changes in the proliferation zone and/or the hypertrophic zone [8,21-23]. In this study, significant differences were found in PCNA- and type X collagen-positive cell numbers between the high-intensity and low-intensity groups. Increased these cell numbers indicate the activity of cell proliferation and hypertrophy in the growth plate. Troib et al. [6] reported that the mRNA expression of type $\mathrm{X}$ collagen increased by treadmill exercise which is comparable to the high-intensity and high frequency and shortduration exercise in this study, whereas we observed no changes in the cell numbers expressing type $\mathrm{X}$ collagen. Our 
findings showed that the protein levels for the type $\mathrm{X}$ collagen remains unchanged after high-intensity and high-frequency and short-duration exercise. Moreover, the thickness of the growth plate has increased in all high-intensity exercise groups except for the HHS group. This result was detected in the group seen both cell proliferation and hypertrophy. Give these results, a thinning of the growth plate must result from the activity of cell proliferation and hypertrophy. On the other hand, cell proliferation and hypertrophy of the HHS group had no change in the thickness of the growth plate was not significantly altered unlike the other high-intensity groups; Therefore, an increase in the thickness of the growth plate may be crucial for increases in both cell proliferation and hypertrophy.

The thickness of the growth plate reflects bone growth [22,24-27]. Many animal studies have reported that increased proliferative chondrocytes contribute to longitudinal growth of the bone $[7,21,22]$ and that the thickness of the hypertrophic zone correlates with the rate of bone growth [27-30]. Moreover, increased chondrocytes in the growth plate, especially density of hypertrophic chondrocyte, are typically reflected in limb elongation [29]. In our study, high-intensity exercise increased all of the thickness of the growth plate, chondrocyte proliferation, and hypertrophy compared to lowintensity exercise; and these results can be explained by the effect on promoting bone growth after high-intensity exercise. Excessive mechanical stress inhibits bone growth, although the thickness of the growth plate and chondrocyte numbers increased in intensity-dependent in this study [3,31-33]. The speed of $18 \mathrm{~m} / \mathrm{m}$ applied in this study corresponds to lactate threshold and the critical speed for aerobic capacity, an intense exercise [34]. Hagihara et al. showed that bone density significantly decreased after different duration (for 30 or 180 $\mathrm{min}$ ) of moderate intensity exercise in rats [35]. Furthermore, they examined the effect of exercise in 4-7 $\mathrm{d}$ a week and revealed exercise in 4 or $5 \mathrm{~d}$ a week is the best for increasing bone density [36]. Accordingly, factors other than intensity such as frequency or duration may have a negative effect on bone growth.

The low-intensity exercise has led to low activities of chondrocytes and no change in the thickness of the growth plate compared to the high-intensity exercise. The exercise speed of $10 \mathrm{~m} / \mathrm{min}$ corresponds to walking in the C57BL/6J mice and then $8 \mathrm{~m} / \mathrm{min}$ in this study regarded as low-intensity walking exercise [18]. Our results indicate that walking exercise did not affect the chondrocytic activities and the thickness of the growth plate.

Through the sequential process of cell proliferation, hypertrophy, and eventually a vascular invasion and the deposition of the bone matrix, chondrocytes continually is being replaced by bone [3]. Then, we observed the bone metabolism in the primary spongiosa to reveal whether chondrocyte activities in the growth plate contribute to increase in the epiphyseal cancellous bone or not. As a result, ALP activities, the osteoblast marker, showed no difference among the groups, but TRAP activities, the osteoclast marker, significantly increased by high-intensity and low-frequency and long duration exercise. The endochondral ossification is the process that results in an ossification through osteoclast and osteoblast activities and therefore bone resorption by osteoclast plays important role in endochondral ossification [37]. Increased TRAP activities by high-intensity and low-frequency and long-duration exercise imply the promotion of bone metabolism from the growth plate to the primary spongiosa. However, the bone metabolism in the primary spongiosa promoted in not all high-intensity groups that cell activities increased in the growth plate. In contrast to our results, Niehoff et al. reported that the thickness of the growth plate and the proliferative and hypertrophic zones decreased after wheel running corresponding to intense exercise for mice [7]. These contradictory results can be explained by the calcified speed in the primary spongiosa exceeding chondrocytic activation; that is, the loading stimulation of their study may have more influence on bone metabolism than chondrocyte activities in the growth plate. Therefore, the chondrocyte activities in the growth plate and bone metabolism may differ depending on loading stimulation.

The high-intensity running exercise has promoted cell proliferation and hypertrophy in the growth plate, and eventually increased the thickness of the growth plate. Additionally, we revealed that the osteoclast activities in the primary spongiosa promoted by the high-intensity and lowfrequency and long-duration exercise. A potential weakness of the study is that the growth in length of long bones itself was not measure directly, although the thickness the growth plate was evaluated. Our results suggest that chondrocyte activity and bone metabolism promote in high-intensity and lowfrequency and long-duration in young mice, and these findings may shed light on the underlying of the promotion of the longitudinal bone growth by exercise.

\section{Acknowledgement}

This study was supported in part by Japan Society for the Promotion of Science (JSPS) KAKENHI Grant Number 25702032.

\section{References}

1. Lui JC, Nilsson O, Baron J. Growth plate senescence and catch-up growth. Endocr Dev 2011; 21: 23-29.

2. Robling AG, Duijvelaar KM, Geevers JV, Ohashi N, Turner $\mathrm{CH}$. Modulation of appositional and longitudinal bone growth in the rat ulna by applied static and dynamic force. Bone 2001; 29: 105-113.

3. Mirtz TA, Chandlerb JP, Eyers CM. The effects of physical activity on the epiphyseal growth plates: a review of the literature on normal physiology and clinical implications. J Clin Med Res 2011; 3: 1-7.

4. Ballock RT, O'Keefe RJ. Physiology and pathophysiology of the growth plate. Birth Defects Res C Embryo Today 2003; 69: 123-143. 
5. Nilsson O, Marino R, De Luca F, Phillip M, Baron J. Endocrine regulation of the growth plate. Horm Res 2005; 64: 157-165.

6. Troib A, Guterman M, Rabkin R, Landau D, Segev Y. Endurance exercise and growth hormone improve bone formation in young and growth-retarded chronic kidney disease rats. Nephrol Dial Transplant 2016; 31: 1270-1279.

7. Niehoff A, Kersting UG, Zaucke F, Morlock MM, Brüggemann GP. Adaptation of mechanical, morphological, and biochemical properties of the rat growth plate to dose dependent voluntary exercise. Bone 2004; 35: 899-908.

8. Nyska M, Nyska A, Swissa-Sivan A, Samueloff S. Histomorphometry of long bone growth plate in swimming rats. Int J Exp Pathol 1995; 76: 241-245.

9. Pescatello LS, Riebe D, Arena R, Paul D: ACSM's guidelines for exercise testing and prescription (9th Ed.). Baltimore Lippincott Williams \& Wilkins 2014.

10. Grimston SK, Willows ND, Hanley DA. Mechanical loading regime and its relationship to bone mineral density in children. Med Sci Sports Exerc 1993; 25: 1203-1210.

11. Yoshimura N. Exercise and physical activities for the prevention of osteoporotic fractures: A review of the evidence. Nihon Eiseigaku Zasshi 2003; 58: 328-337.

12. Umemura Y, Ishiko T, Yamauchi T, Kurono M, Mashiko S. Five jumps per day increase bone mass and breaking force in rats. J Bone Miner Res 1997; 12: 1480-1485.

13. Kato T, Terashima T, Yamashita T, Hatanaka Y, Honda A, Umemura Y. Effect of low-repetition jump training on bone mineral density in young women. J Appl Physiol 2006; 100: 839-843.

14. Laor T, Wall EJ, Vu LP. Physeal widening in the knee due to stress injury in child athletes. AJR Am J Roentgenol 2006; 186: 1260-1264.

15. Baechle TR, Earle RW. Essentials of strength training and conditioning (2nd Ed.). Human Kinetics, Champaign, IL 2000 .

16. Wallace JM, Rajachar RM, Allen MR, Bloomfield SA, Robey PG, Young MF, Kohn DH. Exercise-induced changes in the cortical bone of growing mice are bone and gender-specific. Bone 2007; 40: 1120-1127.

17. Suominen H, Kiiskinen A, Heikkinen E. Effects of physical training on metabolism of connective tissues in young mice. Acta Physiol Scand 1980; 108: 17-22.

18. Sturgeon K, Schadler K, Muthukumaran G, Ding D, Bajulaiye A, Thomas NJ, Ferrari V, Ryeom S, Libonati JR. Concomitant low-dose doxorubicin treatment and exercise. Am J Physiol Regul Integr Comp Physiol 2014; 307: 685-692.

19. Wu J, Wang XX, Higuchi M, Yamada K, Ishimi Y. High bone mass gained by exercise in growing male mice is increased by subsequent reduced exercise. J Appl Physiol 2004; 97: 806-810.
20. Kawamoto T, Kawamoto K. Preparation of thin frozen sections from nonfixed and undecalcified hard tissues using Kawamoto's film methods (2012). Methods Mol Biol 2014; 1130: 149-164.

21. Huang TH, Yang RS, Hsieh SS, Liu SH. Effects of caffeine and exercise on the development of bone: A densitometric and histomorphometric study in young wistar rats. Bone 2001; 30: 293-299.

22. Swissa-Sivan A, Simkin A, Leichter I, Nyska A, Nyska M, Statter M, Bivas A, Menczel J, Samueloff S. Effect of swimming on bone growth and development in young rats. Bone Miner 1989; 7: 91-105.

23. Reich A, Jaffe N, Tong A, Lavelin I, Genina O, Pines M, Sklan D, Nussinovitch A, Monsonego-Ornan E. Weight loading young chicks inhibits bone elongation and promotes growth plate ossification and vascularization. J Appl Physiol 2005; 98: 2381-2389.

24. Forwood MR, Parker AW. Effects of exercise on bone growth: mechanical and physical properties in the rat. Clin Biomech 1987; 2: 185-190.

25. Kember NF. Comparative patterns of cell division in epiphyseal cartilage plates in the rat. J Anat 1972; 111: 137-142.

26. Roach HI, Mehta G, Oreffo RO, Clarke NM, Cooper C. Temporal analysis of rat growth plates: cessation of growth with age despite presence of a physis. J Histochem Cytochem 2003; 51: 373-383.

27. Wilsman NJ, Farnum CE, Leiferman EM, Fry M, Barreto C. Differential growth plates as a function of multiple parameters of chondrocytic kinetics. J Orthop Res 1996; 14: 927-936.

28. Farnum, Cornelia E, Wilsman. Converting a differentiation cascade into longitudinal growth: stereology and analysis of transgenic animals as tools for understanding growth plate function. Curr Opinion Orthop 2001; 12: 428-433.

29. Hunziker EB, Schenk RK. Physiological mechanisms adopted by chondrocytes in regulating longitudinal bone growth in rats. J Physiol 1989; 414: 55-71.

30. Kember NF. Comparative patterns of cell division in epiphyseal cartilage plates in the rabbit. J Anat 1985; 142: 185-190.

31. Stokes IA, Aronsson DD, Dimock AN, Cortright V, Beck $\mathrm{S}$. Endochondral growth in growth plates of three species at two anatomical locations modulated by mechanical compression and tension. J Orthop Res 2006; 24: 1327-1334.

32. Stokes IA, Clark KC, Farnum CE, Aronsson DD. Alterations in the growth plate associated with growth modulation by sustained compression or distraction. Bone 2007; 41: 197-205.

33. Villemure I, Stokes IA. Growth plate mechanics and mechanobiology. A survey of present understanding. J Biomech 2009; 42: 1793-1803. 
34. Billat VL, Mouisel E, Roblot N, Melki J. Inter- and intrastrain variation in mouse critical running speed. J Appl Physiol 2005; 98: 1258-1263.

35. Hagihara Y, Nakajima A, Fukuda S, Goto S, Iida H, Yamazaki M. Running Exercise for short duration increase BMD of loaded long bone in young growing rats. Tohoku J Exp Med 2009; 219: 139-143.

36. Hagihara Y, Fukuda S, Goto S, Iida H, Yamazaki M, Moriya H. How many days per week should rats undergo running exercise to increase BMD? J Bone Miner Metab 2005; 23: 289-294.

37. Fujii K, Inoue H. Biology of bone and cartilageApplication of basic science for practice medicine. Kanehara \& Co., Ltd., Tokyo, Japan 2002.

\section{*Correspondence to}

Hideki Moriyama

Life and Medical Sciences Area

Health Sciences Discipline

Kobe University

Hyogo

Japan 